\title{
Characterisation of Internal Flow Conditions in GDI Injectors by Means of Spray-Hole-Individual Mass Flow Rate and Momentum Flux Measurements
}

\author{
Maximilian Miller ${ }^{* 1}$, Philippe Leick ${ }^{1}$, Ralf Köhler ${ }^{1}$, Maximilian Kuhnhenn ${ }^{1}$, Ingo Samerski ${ }^{1}$, \\ Grazia Lamanna², Bernhard Weigand ${ }^{2}$ \\ ${ }^{1}$ Powertrain Solutions, Robert Bosch GmbH, Schwieberdingen, Germany \\ ${ }^{2}$ Institute of Aerospace Thermodynamics, University of Stuttgart, Germany \\ *Corresponding author email: maximilian.miller@gmx.de, philippe.leick@de.bosch.com
}

\begin{abstract}
High-pressure multi-hole injectors for gasoline direct injection (GDI) engines allow a flexible optimisation of the individual spray hole properties to specific engines and combustion strategies. One degree of freedom within this spray targeting process is the mass flow rate of each spray plume, which is mainly controlled by the corresponding spray hole diameter. As a first approximation, it can safely be assumed that the flow rate scales with the spray hole crosssectional area, but due to the complex flow pattern within the valve seat, some deviations are likely to occur. This was investigated using four specially designed real-size research injectors with two almost identical spray holes - except for their diameters, one of them constant and the other variable. Combining the results of spray-hole-individual mass flow rate and spray force measurements, the spray-hole-individual discharge coefficients can be separated into area and velocity coefficients. Under the choked flow conditions typical of GDI, the discharge and area coefficients increase while the velocity coefficients decrease as spray holes become smaller. At the same time, the flow inside the unaltered spray hole is affected as well, although the discharge coefficient remains constant because of reciprocal trends between the area and velocity coefficients.
\end{abstract}

\section{Keywords}

mass flow, jet force, gasoline direct injection, discharge coefficient, effective velocity/area

\section{Introduction}

The multi-hole design commonly used for GDI injectors grants a high degree of freedom for the adaptation and optimisation of the spray targeting to the specific requirements of a given engine and its associated combustion strategy. In principle, the geometric parameters that control the internal flow conditions can be chosen individually for each spray hole; in practice, the most commonly varied parameters are the hole directions and diameters. The "ideal" injector generates a spray that favours rapid mixing of the injected fuel with the ambient air and does not deposit significant amounts of fuel on any surface within the combustion chamber [1]. However, given the complexity of the flow in the valve seat and the difficulty of measuring spray properties separately for each plume, much of the optimisation has to happen empirically and based on computational fluid dynamics (CFD) studies. Of course, the total flow rate is routinely measured but the contribution of each hole to this result is usually not known from experiments. To retrieve individual mass flows from each spray hole, and to separate the discharge coefficient into a velocity and an area coefficient, an approach that has been suggested and demonstrated by Payri and co-workers [2] is to deduce the spray-hole-individual flow rates from momentum flux measurements normalised by the total flow rate. The assumption that then needs to be made is that the flow through all holes is relatively similar. This approximation is well justified for typically symmetric layouts of diesel injectors but might no longer be accurate in a typical GDI situation. Combining spray-hole-individual mass flow rate measurements, obtained from our recently 
presented extension of the hydraulic pressure rise method [3], with equally spray-hole-individual momentum flux measurements overcomes this limit. It makes a detailed characterisation of the bulk flow properties in highly asymmetric injectors possible. To the best knowledge of the authors, this work is the first one in which this experimental approach was used on a hole-tohole basis and on GDI injectors.

\section{Material and methods}

Four specially designed generic research injectors with two spray holes located on opposite sides of the injector tips formed the basis of this investigation. All spray holes are identical with respect to their inclination, length, moderate divergent conicity and counterbore dimensions. One spray hole of each injector variant (Var\#1-Var\#4) is constant in diameter $\left(D_{1}\right)$ as well, whereas the second spray hole diameter $\left(D_{2}\right)$ is variable (Table 1). The spread of $D_{2}$ is significantly larger than the range typically covered in GDI applications but this extreme variability was expected to highlight subtle differences in flow conditions. Due to its proven compatibility with the spray force test rig used, all measurements were carried out with diesel calibration fuel, but it was made sure that the highly turbulent and choked flow regimes typical of GDI were reached.

Table 1. Diameters of the spray holes at their narrowest point and corresponding cross-sectional area ratios.

\begin{tabular}{c|ccc}
\hline & $D_{1}(\mu \mathrm{m})$ & $D_{2}(\mu \mathrm{m})$ & $A_{1} / A_{2}(-)$ \\
\hline Var\#1 & 210 & 210 & 1 \\
Var\#2 & 210 & 170 & 1.53 \\
Var\#3 & 210 & 130 & 2.61 \\
Var\#4 & 210 & 90 & 5.44
\end{tabular}

\section{Mass flow rate measurements}

In order to determine spray-hole-individual mass flow rates, two different measurement setups were used. Both are based on the measurement principle of Zeuch [4], in which the liquid is injected into a closed measurement chamber of volume $V$ pre-filled with pressurised liquid and the corresponding sharp back pressure rise $d p_{b} / d t$ is used to calculate the rate of injection $d m / d t$. Assuming an adiabatic and isochoric process and expressing the ratio of liquid bulk modulus $B$ and liquid density $\rho$ by the speed of sound $c=\sqrt{B / \rho}$, the mass flow rate is expressed by

$$
\frac{d m}{d t}=\frac{V}{c^{2}} \frac{d p_{b}}{d t} .
$$

The speed of sound $c$, as the only required fuel-, temperature- and pressure-dependent quantity, can be determined directly [5]. For this purpose, acoustic pulse runtime measurements over a known travel distance are performed inside the measurement chamber with an ultrasonic sensor, making calibration procedures or the use of equations of state unnecessary. Between successive injections, the backpressure level is controlled by activating a drain valve. Studying the mass flow behaviour at different back pressure levels enables hydraulic characterisation of the internal flow conditions. Once the flow is choked due to cavitation, which typically occurs in multi-hole GDI injectors [6], the mass flow rate becomes independent of back pressure and the rise of back pressure due to the injection no longer distorts the measurement result. The commercially available mass flow rate meter HDA by Moehwald $\mathrm{GmbH}[6,7]$ was used to determine the time-resolved, total mass flow rate $d m / d t$ within this study. To split the total mass flow rate into the separate contributions of the two individual spray holes $\left(d m / d t=d m_{1} / d t+d m_{2} / d t\right)$, a recently introduced measurement setup featuring a mechanical separator guiding the flows into separate chambers of identical volume 

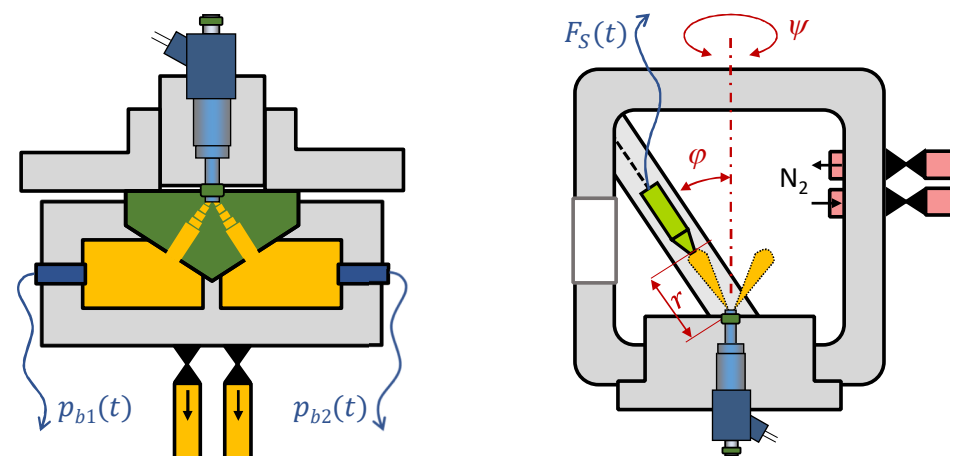

Figure 1. Schematics of relative mass flow rate and jet force measurement test rigs.

was used (Figure 1, left). Assuming an equal speed of sound $c$ inside both chambers, a relative mass flow rate can be calculated by the ratio of back pressure increases only. A detailed description of this setup and the data reduction schemes applied depending on the governing flow conditions can be found in [3]. For each operating point, the total and the relative mass flow rates were averaged over the quasi-steady phase of 200 injections.

\section{Jet Force Measurements}

Spray force or momentum flux measurements are well established in research and development of diesel injectors $[2,8]$ and have been used sporadically to characterise high-pressure gasoline sprays $[9,10]$. Figure 1 (right) shows a sketch of the test rig used within this project; a more detailed description can be found in previous publications [11-13], the addition of a fixture for GDI injectors being the only modification. The fuel is injected into a pressure vessel that can be filled with room temperature nitrogen at pressures up to $2.3 \mathrm{MPa}$ in order to match gas densities in the engine at the moment of injection. The force exerted by the jet on a welldefined flat impact plate is measured with a calibrated piezo-electric pressure sensor. The transducer is mounted on a rail and can be positioned at different distances $r$ from the injector axis; the inclination of the rail ( $\varphi$, elevation angle) is also adjustable, and the whole chamber can be rotated around the fixed injector ( $\psi$, side angle). For each position of the transducer, temporally resolved force histories of 20 injections were averaged and recorded; the shot-toshot fluctuations are too high for meaningful interpretations of data from a single injection. The spatial dependency of spray structure is studied by moving the sensor through the plumes at constant distance $r$ from the injector axis.

The force exerted on the pin surface is a sum of the contributions of droplet impacts and dynamic pressure due to the entrained gas flow within the spray [8]. The exact value depends on the deflection angle of the droplets and on the flow field surrounding the target plate and it is thus difficult to accurately measure the "true" momentum flux at any given sensor position, but the evolution of the measurement results depending on the operating parameters or the injector geometry can be tracked reliably.

For the purpose of this work, it is important to measure the full integrated momentum flux in each spray plume. With narrow diesel spray plumes, when the sensor is located close to the spray hole outlet and an impact plate of typical size (diameter $1-2 \mathrm{~mm}$ ) is used, it can safely be assumed that the jet cross section is much smaller than the target surface and that the full momentum flux is thus collected. For the much wider gasoline spray plumes this is no longer the case. In principle, larger target plates could be used, but this has been shown to increase the confounding effect of pressure waves propagating along the target surface [14] and would also make it likely that more than one plume hits the target in a multi-hole spray. However, the full momentum flux $F_{\text {total }}(r, t)$ can always be reconstructed based on spatial force profiles $F_{S}(r, \varphi, \psi, t)$, which in themselves already are integrals of the momentum flux over the target 

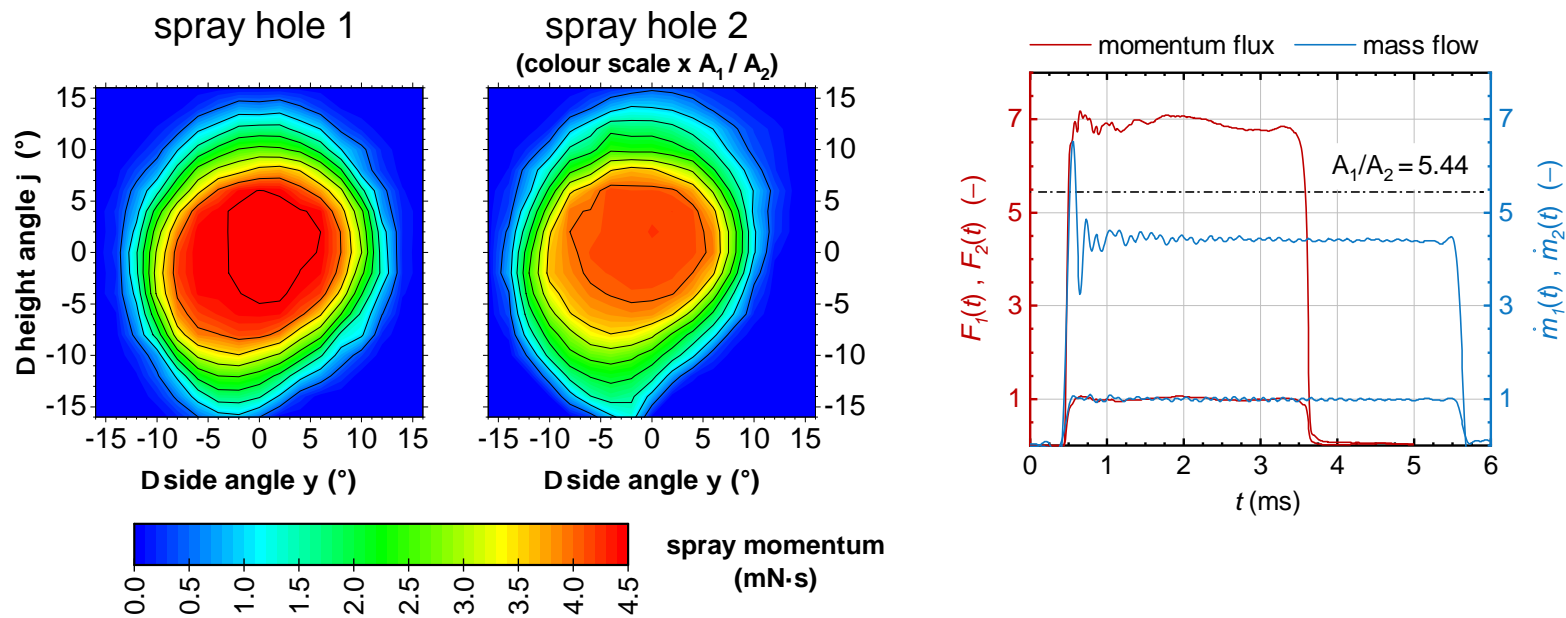

Figure 2. Left: spatial spray momentum $\left(=\int F_{S}(t) d t\right)$ distribution for the injector Var\#4 with a geometric crosssectional area ratio of $A_{1} / A_{2}=5.44$ at $p_{i n j}=30$ and $p_{a m b}=0.5 \mathrm{MPa}$ (note the amplified colour scale for the smaller spray hole 2). Right: Corresponding time-resolved spray force and mass flow rate normalised to the values obtained from the smaller hole 2 during the quasi-steady phase of the injection (injection durations differ between spray force and mass flow rate measurements).

surface $A_{T}$,

$$
F_{\text {total }}(r, t)=1 / A_{T} \int F_{S}(r, \varphi, \psi, t) d \varphi d \psi \approx \Delta \varphi \Delta \psi / A_{T} \sum F_{S}(r, \varphi, \psi, t),
$$

where $\Delta \varphi$ and $\Delta \psi$ are the step sizes inside the force and momentum structures (Figure 2) and expressed in the same units than the sensor dimension. The best approximation of the integral in Eq. 2 would be obtained with small steps and a very small sensor, but the cost of a small sensor would be a lower signal to noise ratio. For this work, the commonly used impact plate with a $2 \mathrm{~mm}$ diameter was assumed to result in the best compromise between the competing criteria and thus selected. The measurements were carried out at a distance of $r=5 \mathrm{~mm}$ from the injector axis, which is almost as close as possible given the injector tip diameter of $6 \mathrm{~mm}$.

\section{Theoretical background}

In a spray hole of cross-sectional area $A$, which the liquid does not necessarily occupy fully (ratio $C_{A}$ ), and where it reaches a bulk velocity given as a fraction of the Bernoulli velocity $\left(u=c_{V} u_{B}, u_{B}=\sqrt{2\left(p_{i n j}-p_{a m b}\right) / \rho}\right)$, the mass and momentum fluxes integrated over the cross section yield the mass flow rate and spray force and can be written as

$$
\begin{aligned}
& \frac{d m}{d t}=\rho c_{D} A u_{B}=\rho\left(c_{A} A\right)\left(c_{V} u_{B}\right) \\
& F=\rho\left(c_{A} A\right)\left(c_{V} u_{B}\right)^{2}=2 c_{A} c_{V}^{2}\left(p_{i n j}-p_{a m b}\right) A .
\end{aligned}
$$

It then becomes straightforward to calculate $c_{A}$ and $c_{V}$ from these two equations and corresponding measurement results assuming a homogeneous flow, e.g. neglecting the fact that the coefficients are average values over the spray hole cross section and that $\overline{C_{A} C_{V}}=\overline{C_{A}} \cdot \overline{C_{V}}$ and $\overline{C_{A} C_{V}^{2}}=\overline{C_{A}} \cdot{\overline{C_{V}}}^{2}$ can thus not generally be assumed. For gasoline direct injection, the flow is likely to be choked due to cavitation within the spray holes. In corresponding models [15,16], the narrowest contraction (vena contracta) occurs when the pressure drops to the vapour pressure $p_{V}$ and the liquid then occupies a fraction $c_{C}$ of the available cross section, leading to slightly different expressions for the mass flow rate and momentum flux at the hole outlet:

$$
\frac{d m}{d t}=\rho\left(c_{C} A\right) \sqrt{\frac{2\left(p_{i n j}-p_{V}\right)}{\rho}}=\rho\left(c_{C} A\right) \sqrt{K} u_{B}
$$




$$
2 c_{C}^{2}\left(p_{i n j}-p_{V}\right) A \leq F \leq 2\left(c_{C} A\right)\left(p_{i n j}-p_{V}\right)-\left(p_{a m b}-p_{V}\right) A .
$$

Equation 6 is bound by slug-flow (left) and zero-wall-shear (right) assumptions between the vena contracta and the outlet and $K=\left(p_{i n j}-p_{V}\right) /\left(p_{i n j}-p_{a m b}\right)$ in Eq. 5 is Nurick's cavitation number. In analogy to Eqs. $3-4$, however, the outlet conditions can still be described with effective cross sections and velocities that yield the same mass flow and momentum flux as the experimentally measured values and this approach will be used throughout the remainder of the paper.

\section{Results and discussion}

For the purpose of hydraulic characterisation, a combination of injection pressures ranging from 2.0 up to $30.0 \mathrm{MPa}$ against backpressures from 0.5 to the maximum of $2.0 \mathrm{MPa}$ achievable in the relative mass flow rate test rig were investigated. Lower back pressures than $0.5 \mathrm{MPa}$ are problematic because shear cavitation might develop between the incoming jet and the ambient liquid. Plotting the normalised mass flow over $1 / \sqrt{K}$ makes it possible to group measurements from different injection pressure levels and immediately reveals where the transition from unchoked to choked flow occurs. As an example, this is shown on the left side of Figure 3 for Var\#1 and Var\#4, where it can be seen that the transition occurs simultaneously for both spray holes. Similar results are obtained for the intermediate Var\#2 and Var\#3 but omitted in order not to overburden the diagram. All experiments described in the subsequent sections of the paper were carried out at realistic values of ambient and injection pressure and are well within the choked flow regime $(1 / \sqrt{K} \gg 0.8)$. The comparison with mass flow rate results obtained with n-heptane as a more common and realistic gasoline surrogate instead of diesel calibration oil (Figure 5, left) shows that, at the high pressure differences relevant for GDI, the flow conditions are very similar for both liquids in terms of the contraction coefficient $c_{C}$. It is thus legitimate to facilitate the jet force measurements using the diesel calibration oil wellestablished for this technique and to draw general conclusions from comparisons of the mass flow rate and momentum flux measurements carried out under nearly identical experimental conditions.

The right side of Figure 3 shows the spray force measurement results obtained for Var\#4, e.g. the injector with the most extreme difference between the two spray holes. Similar results are obtained for the other three injectors. The signals are integrated over the spatial profile according to Eq. 2 and averaged over the quasi-steady phase of injection. The forces exerted on the sensor by both spray plumes can be seen to be proportional to the pressure difference
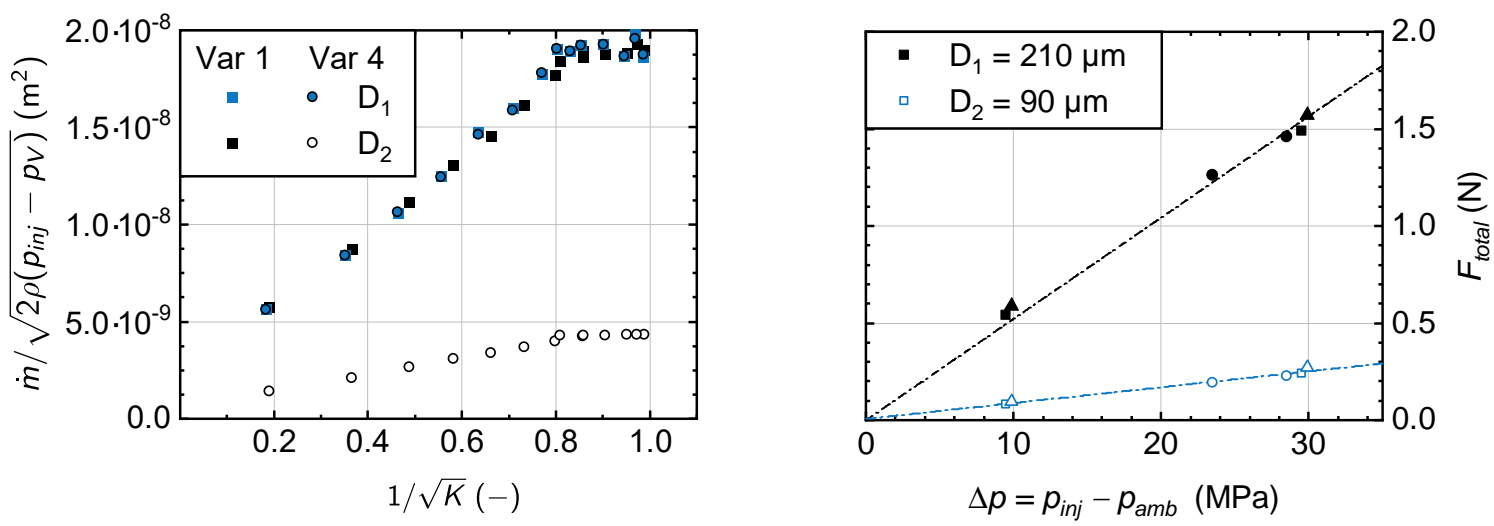

Figure 3. Increase of normalised flow rate against reciprocal square root of Nurick's cavitation number (left) and increase of measured spray force with pressure difference (right), along with linear fits to the data (at backpressures of $0.1(\triangle), 0.5(\square)$ and $1.5 \mathrm{MPa}(\bigcirc))$. The slopes of the fit lines summarise the momentum flux measurements; both flow rate and force are time-averaged over the quasi-steady phases of the injections. 

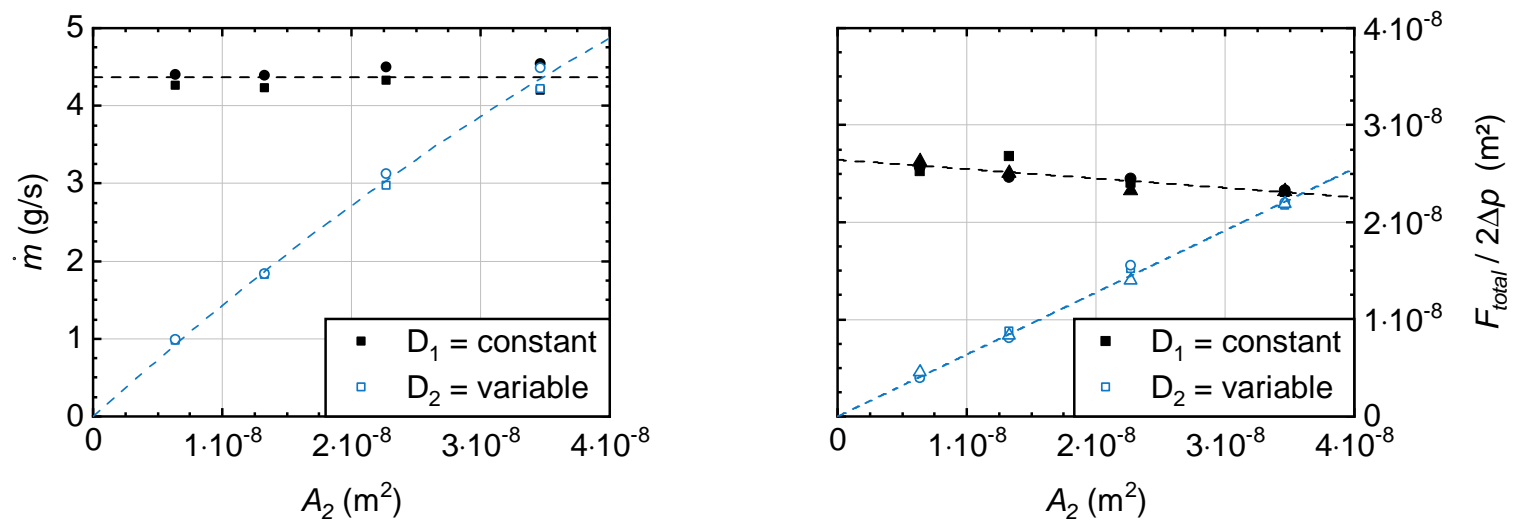

Figure 4. Mass flow rate against spray hole cross section (left) and normalised spray force against spray hole cross section (right), data points with 0.1 ( $\triangle$, jet force only), $0.5(\square)$ and $1.5 \mathrm{MPa}(\bigcirc)$ back/ambient and $30 \mathrm{MPa}$ injection pressure. The dotted lines are fits to the data.

$p_{i n j}-p_{a m b}$. The dependence on ambient pressure contradicts the slug-flow assumption and agrees well with the zero-wall-shear model, although the range of back pressures is too small to rule out unchoked flow based on spray force measurements alone.

For operating conditions where both mass flow rates and spray forces are available and signalto-noise ratios are high - e.g. at injection pressures of $30 \mathrm{MPa}$ - the experimental results are plotted over the cross section $A_{2}$ of the variable spray hole (Figure 4). It is apparent that the mass flow through the unaltered spray hole (1) does not depend on the size of the variable spray hole (2) and that the discharge coefficient $c_{D 1}$ is thus constant. As expected, the flow through the variable size spray hole increases with available cross-sectional area. However, there is a slight deviation from linear behaviour; in fact, the discharge coefficient increases from about 0.56 to almost 0.70 as the hole diameter decreases from 210 to $90 \mu \mathrm{m}$, which agrees well with the systematic CFD investigations of Hellmann et al. in symmetric injectors [17]. The spray force measurements reveal a different trend: the force of the variable-size spray hole increases linearly with the available cross section, whereas the force of the constant spray hole decreases as the variable spray hole becomes larger, which suggests some kind of "competition for a limited resource" (e.g. pressurised liquid) between the two spray holes.

Given that both mass flow rate and momentum flux depend linearly on flow area $\left(c_{A} A\right)$ but that mass flow scales with velocity $\left(c_{V} u_{B}\right)$ and momentum flux with the square of velocity, a relatively straightforward interpretation is as follows:

- For the constant-size spray hole, velocity increases and flow area decreases as the variable spray hole becomes smaller. A possible explanation is that velocity losses due to wall-friction decrease as a larger fraction of the hole is occupied by vapour.

- For the variable spray hole, the reverse is true: As its diameter becomes smaller, area utilisation by the liquid phase increases and its bulk velocity decreases, possibly due to higher circumference to cross section ratio and thus to increased importance of wall friction.

The latter trends regarding the changes in area utilisation and bulk velocity inside the altered spray hole agree well with results from CFD-studies by $\mathrm{Li}$ and co-workers [18]. Since the absolute values of measured momentum flux need to be interpreted with caution, they are not used to retrieve area and velocity coefficients directly. However, the trends seen in Figure 4 can be "locked" to a plausible starting value. There is ample evidence in the literature that the bulk flow velocity in GDI spray holes is of the order of $85-90 \%$ of Bernoulli velocity [19-22]. We have thus used $c_{V 1}=0.89$ as an initial value for the unaltered spray hole of the injector with two identical spray holes (Var\#1, spray hole 1) and calculated how the area and velocity 

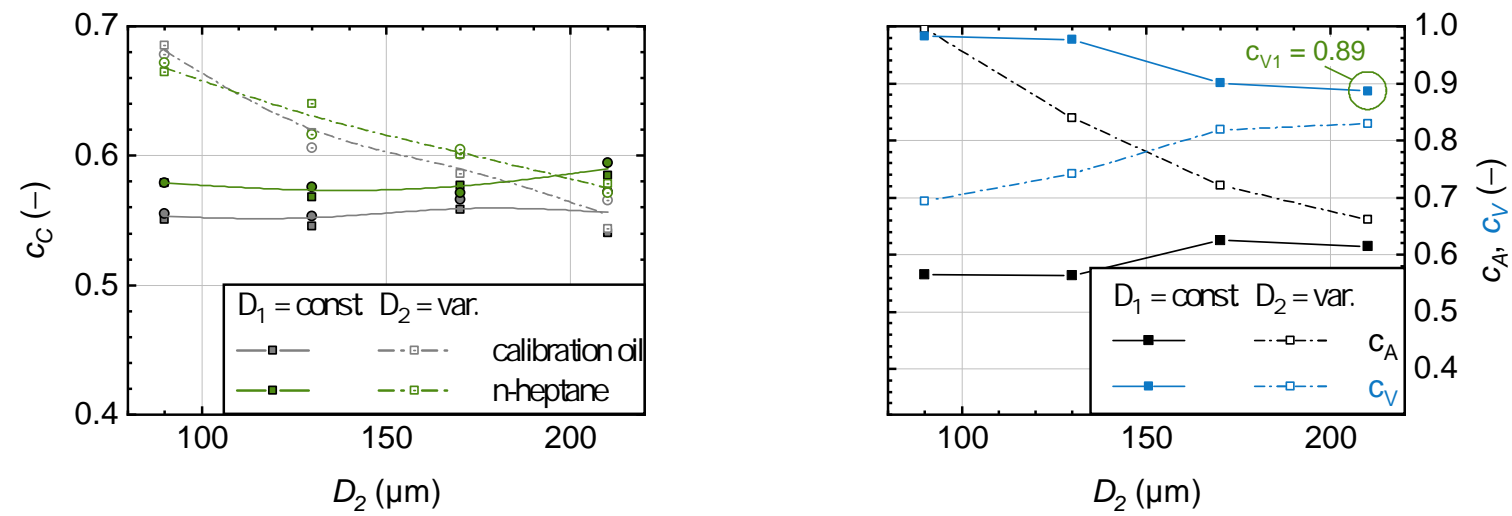

Figure 5. Contraction coefficients $c_{C}$ from flow rate measurement with n-heptane (green) and diesel calibration oil (grey) at back pressures of $0.5(\square)$ and $1.5 \mathrm{MPa}(\bigcirc)$ (left), area and velocity coefficients, assuming $c_{V 1}=0.89$

for spray hole 1 of Var\#1 (right), $c_{A}$ and $c_{V}$ reference to $p_{\text {inj }}=30 \mathrm{MPa}$ and $p_{\text {amb }}=0.5 \mathrm{MPa}$ (right).

coefficients evolve as the size of the variable hole decreases (Figure 5, right), giving us a quantitative version of the statements made in the previous paragraph.

For example, it is apparent that there is more variability in the area than in the velocity coefficient. There also seems to be more variability in the flow through the variable-size spray hole than in the flow of the constant spray hole. However, the effect in the constant-size spray hole is significant enough to suggest that the flow is not only determined by hole geometry but also strongly influenced by the flow in the valve seat area upstream of the spray hole inlets. Another interesting detail is that, in the variable size hole, the main change occurs as the hole becomes very small; however, for the larger spray hole, the main effect is seen once the opposite spray hole is no longer small and thus no longer has a negligible effect on the inflow conditions.

\section{Conclusions}

Measurements of the mass flow rate and momentum flux can be combined to gain detailed insights about the state of the flow inside a spray hole; more specifically, the results can be interpreted in terms of a bulk flow velocity and an effective area occupied by the liquid flow. For multi-hole injectors where not all of the spray holes are equivalent - a typical situation in gasoline direct injection - corresponding measurements need to be carried out individually for each spray hole. To achieve this, a test rig where the flow rate of each spray hole can be measured separately was previously developed and the jet force technique was adapted to gasoline sprays. In order to isolate the effect of hole size on flow rate and to facilitate the separation of the spray-hole-individual flows, a set of custom-made injectors with only two spray holes was used, but an extension to more realistic configurations is considered to be feasible, as long as the targeting offers sufficient spatial separation between the holes and plumes.

The experimental results presented here reveal not only that the flow inside the spray holes depends strongly on their diameter itself, but also that it is significantly affected by the amount of flow through the opposite spray hole, a surprising result confirmed by high-resolution spray imaging. As the diameter of the variable-size hole increases, the expected slight widening of the spray from the variable-size hole is seen. But there is also a clear change of direction for the spray from the constant-size hole, which moves towards the injector axis as the size of the opposite hole increases, and this strengthens our interpretation that the two nozzle-flows are not independent.

The differences in area utilisation between the two spray holes suggest that, at least in strongly asymmetric layouts such as those investigated here, spray-hole-individual mass flow rates should not be reconstructed based solely on momentum flux measurements gauged with a total mass flow reference, as this requires the assumption that either $c_{A}$ or $c_{V}$ are identical for all 
spray holes. However, this approximation might be appropriate in relatively symmetric injector layouts and has the obvious advantage of not requiring separate mass flow measurements for each spray hole [10].

\section{Nomenclature}

$\begin{array}{llll}A & \text { spray hole cross-sectional area }\left[\mathrm{m}^{2}\right] & A_{T} & \text { target plate cross-sectional area }\left[\mathrm{m}^{2}\right] \\ B & \text { bulk modulus [Pa] } & c & \text { speed of sound [m/s] } \\ c_{A} & \text { area coefficient }[-] & c_{C} & \text { contraction coefficient }[-] \\ c_{D} & \text { discharge coefficient }[-] & c_{M} & \text { momentum coefficient }[-] \\ c_{V} & \text { velocity coefficient }[-] & D & \text { spray hole diameter [m] } \\ F & \text { force or integrated momentum flux }[\mathrm{N}] & K & \text { cavitation number [-] } \\ m & \text { mass }[\mathrm{kg}] & p_{a m b} & \text { ambient pressure }[\mathrm{Pa}] \\ p_{b} & \text { backpressure }[\mathrm{Pa}] & p_{\text {inj }} & \text { injection pressure [Pa] } \\ p_{V} & \text { vapour pressure }[\mathrm{Pa}] & \varphi & \text { elevation angle }\left[{ }^{\circ}\right] \\ \psi & \text { side angle }\left[{ }^{\circ}\right] & r & \text { distance to injector axis }[\mathrm{m}] \\ \rho & \text { liquid density }\left[\mathrm{kg} / \mathrm{m}^{3}\right] & t & \text { time }[\mathrm{s}] \\ u_{B} & \text { velocity according to Bernoulli }[\mathrm{m} / \mathrm{s}] & V & \text { volume }\left[\mathrm{m}^{3}\right]\end{array}$

\section{References}

[1] Duronio F., De Vita A., Alloca L. and Anatone M., 2020, Fuel 265:116948.

[2] Payri R., Garcia J.M., Salvador F.J. and Gimeno J., 2005, Fuel 84:551-561.

[3] Miller M., Kuhnhenn M., Samerski I., Lamanna G. and Weigand B., SAE Technical Paper 2020-01-2123.

[4] Zeuch W., 1961, Motorentechnische Zeitschrift 22(9).

[5] Kuhn U., 2004, German Patent DE 10249754 A1.

[6] Dahlander P., lemmolo D. and Tong Y., SAE Technical Paper 2015-01-0929.

[7] Knorsch T., Rogler P., Miller M. and Wiese W., SAE Technical Paper 2016-01-2200.

[8] Postrioti L., Mariani F. and Battistoni M., 2012, Fuel 98:149-163.

[9] Cavicchi A., Postrioti L., Giovannoni N., Fontanesi S., Bonandrini G. and Di Gioia R., 2017, Fuel 206:614-627.

[10] Cavicchi A., Postrioti L., Berni F., Fontanesi S. and Di Gioia R., 2020, Fuel 263:116657.

[11] Komaroff I. and Melcher K., 1971, Bosch Technische Berichte 3(6).

[12] Winter J., Dittus B., Kerst A., Muck O., Schulz R. and Vogel A., 2004, Proceedings THIESEL, Valencia.

[13] Gramlich R., Leick P., Kaleta J., Miller A., Bossmeyer T., Lamanna G., Schmidt D., Roisman I.V. and Tropea C., 2016, Proceedings of $27^{\text {th }}$ ILASS Europe, Brighton.

[14] Ernst J., 2015, PhD thesis, University of Stuttgart.

[15] Nurick W.H., 1976, Journal of Fluids Engineering 98:681-687.

[16] Schmidt D.P. and Corradini M., 1997, Atomization and Sprays 7(6).

[17] Hellmann R., Jochmann P., Leick P., Stapf K.G., Schünemann E. and Thévenin D., 2018, Proceedings of $14^{\text {th }}$ ICLASS Conference, Chicago, IL.

[18] Li X., Cheng Y., Ji S., Yang X. and Wang L., 2019, Energies 12(3):434.

[19] Knorsch T., Mamaikin D., Leick P., Rogler P., Wang J., Li Z. and Wensing M., SAE Technical Paper 2017-01-2302.

[20] Seibel C., Gartung K., Arndt S. and Weigand B., 2004, Fachtagung Lasermethoden in der Strömungsmesstechnik, Karlsruhe.

[21] Gröger K., Kawaharada N., Klippenstein A. and Dinkelacker F., 2019, Proceedings of 29 $9^{\text {th }}$ ILASS Europe, Paris.

[22] Weber D. and Leick P., 2014, Proceedings of $25^{\text {th }}$ ILASS Europe, Bremen. 